\title{
Eine Messe für Soziales und Pflege
}

\section{Neue Wege der Personalrekrutierung für Sozial- und Gesundheitsunternehmen}

\section{TÜRKAN AYAN UND \\ JÜRGEN ZIEHER}

Prof. Dr. Türkan Ayan ist seit 2007 Professorin für Psychologie an der Hochschule der Bundesagentur für Arbeit in Mannheim. Seit 2011 leitet sie das Mannheimer Teilvorhaben des Verbundforschungsprojektes »BEST WSG - Berufsintegrierte Studiengänge zur Weiterqualifizierung im Sozial- und Gesundheitswesen" der Hochschule der Bundesagentur für Arbeit und der Fachhochschule der Diakonie, Bielefeld.

Tuerkan.Ayan@arbeitsagentur.de

Dr. Jürgen Zieher ist seit 2011 wissenschaftlicher Mitarbeiter im Forschungsprojekt "BEST WSG" an der Hochschule der Bundesagentur für Arbeit in Mannheim. Sein Forschungsschwerpunkt liegt in der Analyse der Rekrutierungs- und Qualifizierungswege von Quereinsteigern für das Sozial- und Gesundheitswesen.

Juergen.Zieher@arbeitsagentur.de

\author{
Eine Umfrage zu der in Mannheim stattfindenden \\ Messe für Sozial- und Pflegeberufe "GoSocial» \\ offenbart Stärken und Schwächen dieser \\ Veranstaltungsform und zeigt, mögliches \\ Entwicklungspotenzial für Veranstalter und Aussteller.
}

Bei der Gewinnung von Auszubildenden gehen der Caritasverband Mannheim und die Agentur für Arbeit Mannheim seit 2011 einen neuen Weg. Sie veranstalten in Zusammenarbeit mit dem Theresienkrankenhaus Mannheim einmal jährlich die »GoSocial «, eine eintägige Messe für Sozial- und Pflegeberufe. Besucher (1) bekommen Einblicke in Pflege- und Kindertageseinrichtungen und können sich in Gesprächen mit geschultem Fachpersonal und Auszubildenden über Ausbildungsund Studienmöglichkeiten informieren sowie mit Shuttle-Bussen zu Praxisorten fahren. Die »GoSocial « richtet sich primär an Jugendliche, ist jedoch auch offen für erwachsene Interessierte, die einen Berufswechsel erwägen. (2)

Im allgemeinen Mittelpunkt des Erkenntnisinteresses standen die Chancen, die eine solche Messeveranstaltung bietet, um Nachwuchskräfte und Quereinsteiger für Sozial- und Pflegeberufe zu gewinnen. Die Ergebnisse sollten den Veranstaltern Anhaltspunkte zur Akzeptanz der »GoSocial « unter den Besuchern und Ausstellern sowie Anregungen für eine noch stärker zielgruppenorientierte Konzeption und Umsetzung der Messe liefern.

\section{Messebesuch allgemein}

Lehrkräfte der Schulen (61 \%) sowie Flyer und Plakate (37\%) stellten die Hauptinformationsquellen vorab zur Messe dar. Fast alle Befragten besuchten die Messe erstmalig (93\% der Nennungen). Unter den Jugendlichen wurde das Interesse an Ausbildungsberufen mit 79 Prozent am häufigsten genannt. Bei den über 18-Jährigen hingegen waren Studienan- gebote $(71 \%)$ ausschlaggebend, gefolgt von Ausbildungsberufen mit über einem Drittel der Nennungen. Freie Antworten lassen in vielen Fällen durch den Besuch auf eine Motivationssteigerung oder eine Bestärkung vorhandener Motivation für die Branche vermuten, insbesondere wird der Informationszuwachs durch den Messebesuch als Grund dafür genannt. Insgesamt wollen immerhin knapp 43 Prozent der Befragten später im Sozialund Gesundheitsbereich arbeiten.

\section{Persönliches Gespräch, Bewerbungsunterlagen, Vorstellungsgespräch}

Die Möglichkeit zu einem persönlichen Gespräch wurde von über der Hälfte der Befragten genutzt (58\%). Eine solche direkte Ansprache fiel den meisten Teilnehmern im Vergleich zu einer Kontaktaufnahme per Telefon oder per EMail eindeutig leichter. Lediglich vier Personen geben an, Bewerbungsunterlagen abgegeben zu haben (im Vergleich: knapp zwei Drittel haben keine Unterlagen abgegeben; $32 \%$ keine Angaben.). Dies korrespondiert mit dem Ergebnis, dass die wenigsten Besucher angeben, im Nachgang an die Messe ein Vorstellungsgespräch vereinbart zu haben $(5$ Personen). Der Großteil (62\%) wiederum hat keine Verabredungen getroffen (über ein Drittel macht keine Angaben).

\section{Gesamtbeurteilung durch Besucher und Aussteller}

Eine klare Mehrheit der Besucher (74 \%) und der Aussteller (76\%) be- 


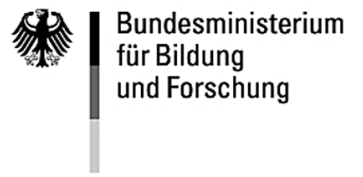

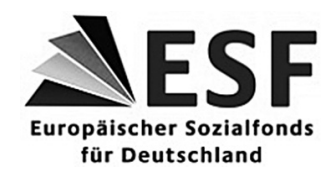

ür Deutschland

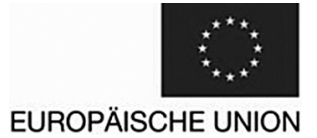

EUROPÄISCHE UNION

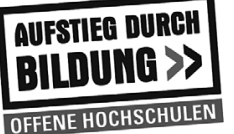

Die für die Befragung ausgewählte Messe "GoSocial« fand am 11. Oktober 2012 zum zweiten Mal statt. Veranstaltungsort war das Theresienkrankenhaus Mannheim. Rund 300 Besucher konnten sich bei acht Unternehmen, drei Fachschulen, einer Hochschule und bei der Berufsberatung der Agentur für Arbeit Mannheim über Ausbildungen im Sozial- und Pflegebereich informieren. Die Teilnehmer und Aussteller der Messe wurden in einem Kooperationsprojekt mit der Hochschule der Bundesagentur für Arbeit befragt. Die Hochschule entwickelte drei Fragebögen: einen für Jugendliche unter 18 Jahren, einen für Erwachsene über 18 Jahre und einen für Messeaussteller. Der Fragenkatalog für den Personenkreis über 18 Jahre zielte auf potenzielle Quereinsteiger, die als arbeitslose Personen, ebenfalls zur Messe eingeladen worden sind. Alle 17 Aussteller beteiligten sich an der Befragung. 150 Besucher füllten ebenfalls einen Fragebogen aus (Rücklaufquote 55 Prozent). Die Befragten sind Großteils weiblich (zwei Drittel) und

Jedoch herrschte am Ende des Messetages bei knapp einem Viertel der Befragten noch Unsicherheit bezüglich der Richtung. Besonders unentschlossen sind hierbei diejenigen, die unter 18 Jahre alt sind. Unsicherheiten können im schulischen Kontext oder aber auch bei der Berufsberatung individuell aufgegriffen und bearbeitet werden, um den Jugendlichen in seiner Entscheidungsphase zu unterstützen.

Bei der niedrigen Zahl an abgegebenen Bewerbungsunterlagen, sind gerade bei den Jugendlichen im Vorfeld mit den Schulen oder der Berufsberatung zu überlegen, ob nicht das Erstellen von Unterlagen und persönliche Einreichen am Messetag stärker angestrebt werden sollte. Auch wurde sowohl von Teilnehmer als auch von Ausstellerseite die Chance, direkt Bewerbungsgespräche zu vereinbaren, noch zu wenig genutzt.

Insgesamt wird bezogen auf die Aspekte der Unsicherheit, der Bewerbungsunterlagen und Bewerbungsgespräche das Potenzial für Nachfassaktionen und Nachsorge gesehen, welches in Kooperation (oder Einzelverantwortung) der Akteure Schule, Unternehmen und Arbeitsagentur umgesetzt werden könnte.

Potenzielle Quereinsteiger fühlten sich insgesamt weniger von diesem, auf Jugendliche fokussierten Veranstaltungsformat angesprochen. Die Zahlen (nur 12 Personen waren 25 Jahre und älter) verdeutlichen, dass die gezielte Ansprache von circa 130 potenziellen Quereinsteigern im Alter von über 25 Jahren durch die Agentur für Arbeit Mannheim nur bedingt erfolgreich war. jünger als 18 Jahre. Der Männeranteil lag bei nicht ganz einem Viertel (Rest: keine Angaben). Von den über 18-Jährigen sind nur zwölf Personen 25 Jahre und älter. Die Befragungen erfolgten im Projekt BEST WSG, einem Teilprojekt zum Quereinstieg in den Sozial- und Gesundheitssektor). Dieses Projekt wird gefördert mit Mitteln des Bundesministeriums für Bildung und Forschung (BMBF) und des Europäischen Sozialfonds. www.bestwsg-hdba.de

Um potenzielle Quereinsteiger - etwa Berufsrückkehrerinnen oder arbeitslose Personen- noch gezielter anzusprechen, sind zielgruppenspezifische Informationsangebote und eine verstärkte Offenheit von Arbeitgebern erforderlich. Denkbar sind neben Informationsveranstaltungen in Pflege- oder Sozialeinrichtungen regelmäßige, auf Erwachsene ausgerichtete Jobbörsen für Sozial- und Pflegeberufe. Dabei sind relevante Arbeitsmarktinstrumente für einen Quereinstieg in Sozial- und Pflegeberufe zu berücksichtigen. Die jeweils 2013 gestartete "Ausbildungs- und Qualifizierungsoffensive Altenpflege « und die »Initiative Erstausbildung junger Erwachsener "bieten interessierten Quereinsteigern umfassendere Fördermöglichkeiten.

Bei der dritten »GoSocial« am 24. Oktober 2013 verzeichneten die Veranstalter mit mehr als 450 Besuchern einen starken Zuwachs an Interessenten. Zahlreiche erwachsene Teilnehmer informierten sich bei Ausstellern über Umschulungen zum Altenpfleger und zum Erzieher oder erkundigten sich bei der Agentur für Arbeit nach Anrechnungsmöglichkeiten ihres im Ausland erworbenen Abschlusses in einem Gesundheits- oder Sozialberuf.

\section{Anmerkungen}

(1) Gemeint sind weibliche und männliche Befragte, sofern nicht explizit gekennzeichnet.

(2) Die dargestellten Ergebnisse sind einem umfassenden Ergebnisbericht entnommen und daraus zusammengefasst. 